\title{
Mean square limit for lattice points in a sphere
}

\author{
by \\ Pavel M. Bleher (Indianapolis, Ind.) and \\ Freeman J. Dyson (Princeton, N.J.)
}

1. Main result. Let

$$
N(R)=\#\left\{n \in \mathbb{Z}^{3}:|n| \leq R\right\}
$$

be the number of integral points inside a sphere of radius $R$ centered at the origin and

$$
F(R)=R^{-1}\left(N(R)-(4 / 3) \pi R^{3}\right) .
$$

We prove in this note the following result:

THEOREM 1.1.

$$
\lim _{T \rightarrow \infty}(T \log T)^{-1} \int_{1}^{T}|F(R)|^{2} d R=K
$$

with $K=(32 / 7)(\zeta(2) / \zeta(3))$.

The three-dimensional case is the most difficult one. A version of Theorem 1.1 is known for a long time for the circle (see [Cra] and [Lan1]) and for the $d$-dimensional ball when $d \geq 4$ (see [Wal]). In [Ble1] a similar statement was proved for any strictly convex (in the sense that the curvature of the boundary is positive everywhere) oval in the plane with the origin inside the oval. Making an analogy to the theory of renormalization group in statistical mechanics we may say that three is the critical dimension for the problem under consideration. Namely, the series of squared Fourier amplitudes of $N(R)$ converges when $d<3$ and diverges when $d \geq 3$ (in fact logarithmically diverges when $d=3$ ). The criticality of $d=3$ is reflected then in the appearance of the log-correction in (1.1).

It is easy to show that

$$
\lim _{T \rightarrow \infty} T^{-1} \int_{1}^{T} F(R) d R=0,
$$


hence Theorem 1.1 implies that

$$
F(R)=\Omega_{ \pm}\left(\log ^{1 / 2} R\right)
$$

(which means that $\limsup _{R \rightarrow \infty}(\log R)^{-1 / 2}( \pm F(R))>0$ ). The estimate $F(R)=\Omega_{-}\left(\log ^{1 / 2} R\right)$ was proved long ago by Szegö [Sze], and Nowak [Now] proved the estimate

$$
F(R)=\Omega_{-}\left(\log ^{1 / 3} R\right)
$$

for any strictly convex domain in $\mathbb{R}^{3}$ with the origin inside the domain. The estimate $F(R)=\Omega_{+}\left(\log ^{1 / 2} R\right)$ is new and it improves the recent result $F(R)=\Omega_{+}(\log \log R)$ by Adhikari and Pétermann [AP] (the authors thank Andrzej Schinzel for calling their attention to the works of Szegö and Adhikari and Pétermann).

See also earlier works [Wal], $[\mathrm{CN}]$ and $[\mathrm{BK}]$ where somewhat weaker estimates were obtained.

As concerns $O$-results, Landau [Lan2] proved that

$$
F(R)=O\left(R^{\theta}\right)
$$

with $\theta=1 / 2$. Vinogradov [Vin1] strengthened this result to $\theta=5 / 14+\varepsilon$, $\forall \varepsilon>0$, and Chen Jing-Run [Che] and Vinogradov [Vin2] to $\theta=1 / 3+\varepsilon$. Recently Chamizo and Iwaniec [CI] strengthened this further to $\theta=7 / 22+\varepsilon$. Randol [Ran] proved the $O$-estimate with $\theta=1 / 2$ for any strictly convex domain in $\mathbb{R}^{3}$ (for ellipsoids it was known before from the work of Landau [Lan2]). Recently Krätzel and Nowak [KN] improved the $O$-estimate of Randol to $\theta=8 / 17+\varepsilon$. For a review and many other results on counting lattice points in multidimensional spheres see [Wal] and [Gro].

Theorem 1.1 can be reformulated in a probability language, namely, that the variance of the random variable $(\log R)^{-1 / 2} F(R)$, assuming that $R$ is uniformly distributed on $[1, T]$, converges to $K>0$ as $T \rightarrow \infty$. An interesting and natural question is then: what is the limiting distribution of $(\log R)^{-1 / 2} F(R)$ (if the latter exists)? We have no answer to this question, but the following simple heuristic argument speaks in favor of a Gaussian limiting distribution.

By formulas (1.12), (1.13) below,

$$
F(R)=-\pi^{-1} \sum_{n \in \mathbb{Z}^{3} \backslash\{0\}} \varphi(2 \pi|n| \delta)|n|^{-2} \cos (2 \pi|n| R)+\varepsilon(R),
$$

where $\varphi(x)$ is a $C^{\infty}$ function fast decreasing at $\infty$ and $\varepsilon(R)$ is an error term. This represents $F(R)$ as an almost periodic function plus an error. We can group terms with commensurate frequencies in (1.2) and rewrite (1.2) as

$$
F(R)=\sum_{\text {square free } k} f_{k}\left(k^{1 / 2} R\right)+\varepsilon(R),
$$


where

$$
f_{k}(t)=-\pi^{-1} k^{-1} \sum_{l=1}^{\infty} l^{-2} r_{3}\left(l^{2} k\right) \varphi\left(2 \pi l k^{1 / 2} \delta\right) \cos (2 \pi l t)
$$

are bounded periodic functions with period 1 and $r_{3}(k)=\#\left\{n \in \mathbb{Z}^{3}\right.$ : $\left.|n|^{2}=k\right\}$. The numbers $k^{1 / 2}$ with square free $k$ (i.e., $k \neq l^{2} k^{\prime}$ with $l>1$ ) are linearly independent over $\mathbb{Z}$ and so the random variables $\left\{\left\{k^{1 / 2} R\right\}, k\right.$ square free $\}$ are asymptotically independent in the limit $T \rightarrow \infty$. Therefore for a fixed $\delta>0$, the limiting distribution of the sum in (1.3) is the same as the limiting distribution of the random series

$$
\xi=\sum_{\text {square free } k} f_{k}\left(\theta_{k}\right),
$$

where $\left\{\theta_{k}\right\}$ are independent random variables uniformly distributed on $[0,1]$.

Observe that

$$
\operatorname{Var} f_{k}\left(\theta_{k}\right)=\int_{0}^{1} f_{k}(t)^{2} d t
$$

is of order of $k^{-2}\left(r_{3}(k)\right)^{2}\left(\varphi\left(2 \pi k^{1 / 2} \delta\right)\right)^{2}$. The series $\sum k^{-2}\left(r_{3}(k)\right)^{2}$ is logarithmically divergent (see Section 2 below), hence $\operatorname{Var} \xi \sim C|\log \delta|^{-1}$. Thus $\xi$ is a series of uniformly bounded independent random variables and the variance of $\xi$ diverges as $\delta \rightarrow 0$. By the Lindeberg theorem this implies that $(\operatorname{Var} \xi)^{-1 / 2} \xi$ converges to a standard Gaussian distribution, so that the limiting distribution of $(\operatorname{Var} F(R))^{-1 / 2} F(R)$ is standard Gaussian as well.

The weakness of this argument is that we took the limit $T \rightarrow \infty$ first and the limit $\delta \rightarrow 0$ second, while in $(1.2) \delta=T^{-1} \lambda(T)$ with $(\log T)^{-1 / 2} \lambda(T) \rightarrow$ 0 , so that $\delta \rightarrow 0$ simultaneously with $T \rightarrow \infty$. This explains why the argument is only heuristic.

For the circle problem Heath-Brown $[\mathrm{H}-\mathrm{B}]$ and Bleher, Cheng, Dyson and Lebowitz [BCDL] proved that the limiting distribution of $(\operatorname{Var} F(R))^{-1 / 2}$ $\times F(R)$ exists and is non-Gaussian with an analytic density decreasing at infinity roughly as $\exp \left(-c x^{4}\right)$.

Proof of Theorem 1.1. Since

$$
N(R)=\sum_{n \in \mathbb{Z}^{3}} \chi(n ; R)
$$

where $\chi(x ; R)$ is the characteristic function of the ball $\{|x| \leq R\}$, the Poisson summation formula implies

$$
N(R)-(4 / 3) \pi R^{3}=\sum_{n}^{\prime} \tilde{\chi}(2 \pi n ; R),
$$


where $\sum_{n}^{\prime}=\sum_{n \in \mathbb{Z}^{3} \backslash\{0\}}$ and $\tilde{\chi}(\xi ; R)$ is the Fourier transform of $\chi(x ; R)$,

$$
\widetilde{\chi}(\xi ; R)=\int_{|x| \leq R} e^{i x \xi} d x=R^{3} \int_{|x| \leq 1} e^{i x R \xi} d x .
$$

Since in addition,

$$
\int_{|x| \leq 1} e^{i x \eta} d x=\pi \int_{-1}^{1} \cos (|\eta| r)\left(1-r^{2}\right) d r=4 \pi|\eta|^{-3}(\sin |\eta|-|\eta| \cos |\eta|),
$$

we obtain

$$
N(R)-(4 / 3) \pi R^{3}=R^{3} \sum_{n}^{\prime} J(2 \pi|n| R),
$$

with

$$
J(t)=4 \pi t^{-3}(\sin t-t \cos t) .
$$

The series in (1.5) is only conditionally convergent. Define for $\delta>0$,

$$
N_{\delta}(R)=\sum_{n \in \mathbb{Z}^{3}} \chi_{\delta}(n ; R)
$$

with

$$
\chi_{\delta}(x ; R)=\delta^{-3} \int_{|y| \leq R} \widetilde{\varphi}\left(\delta^{-1}(x-y)\right) d y,
$$

where $\widetilde{\varphi}(x) \geq 0$ is a $C^{\infty}$ isotropic $\left(\widetilde{\varphi}(x)=\widetilde{\varphi}_{0}(|x|)\right)$ cap with $\widetilde{\varphi}(x)=0$ when $|x| \geq 1$ and $\int_{\mathbb{R}^{3}} \widetilde{\varphi}(x) d x=1$. Then again by Poisson's summation,

$$
N_{\delta}(R)-(4 / 3) \pi R^{3}=R^{3} \sum_{n}^{\prime} \varphi(2 \pi n \delta) J(2 \pi|n| R)
$$

with a convergent series on the right. Put

$$
\delta=T^{-1} \lambda(T),
$$

where $\lambda(T)$ is a slowly increasing function with

$$
\lim _{T \rightarrow \infty} \lambda(T)=\infty \text { and } \lim _{T \rightarrow \infty}(\log T)^{-1 / 2} \lambda(T)=0 .
$$

From (1.9) and (1.6),

$$
\begin{aligned}
& F_{\delta}(R) \\
& \quad:=R^{-1}\left(N_{\delta}(R)-(4 / 3) \pi R^{3}\right) \\
& \quad=-\pi^{-1} \sum_{n}^{\prime} \varphi(2 \pi n \delta)|n|^{-2} \cos (2 \pi|n| R)+O\left(R^{-1} T^{\varepsilon}\right), \quad \forall \varepsilon>0 .
\end{aligned}
$$

We will prove the following two lemmas, from which Theorem 1.1 follows. 
LEMMA 1.2 .

$$
\lim _{T \rightarrow \infty}(T \log T)^{-1} \int_{1}^{T}\left|F(R)-F_{\delta}(R)\right|^{2} d R=0 .
$$

LEMma 1.3.

$$
\lim _{T \rightarrow \infty}(T \log T)^{-1} \int_{1}^{T}\left|F_{\delta}(R)\right|^{2} d R=K>0 .
$$

Proof of Lemma 1.2. We follow the proof of Lemma 4.3 in [Ble2]. To simplify notations we denote by $c$ and $c_{0}$ various constants which can be different in different estimates. We have

so

$$
F_{\delta}(R)-F(R)=R^{-1} \sum_{n}\left(\chi_{\delta}(n ; R)-\chi(n ; R)\right),
$$

with

$$
I \equiv(T \log T)^{-1} \int_{1}^{T}\left|F_{\delta}(R)-F(R)\right|^{2} d R=\sum_{m, n} I(m, n)
$$

$I(m, n)=(T \log T)^{-1} \int_{1}^{T}\left(\chi_{\delta}(m ; R)-\chi(m ; R)\right)\left(\chi_{\delta}(n ; R)-\chi(n ; R)\right) R^{-2} d R$.

Observe that $I(m, n)=0$ unless ||$m|-| n||<2 \delta$ and $|m|,|n|<T+\delta$. In addition, $I(m, n) \leq c(T \log T)^{-1}|n|^{-2} \delta$, for all $m, n$, hence

$$
I \leq c(T \log T)^{-1} \delta \sum_{n:|n| \leq T+\delta}|n|^{-2} \sum_{m:|| m|-| n|| \leq 2 \delta} 1 .
$$

Let us estimate

$$
I_{0}=\sum_{n: T / 2 \leq|n| \leq T} \sum_{m:|| m|-| n|| \leq 2 \delta} 1 .
$$

Observe that $\left.|| m\right|^{2}-|n|^{2}|\leq||m|+|n| \mid 2 \delta \leq 5 T \delta=5 \lambda(T)$, so if $|n|$ is fixed we have not more than $10 \lambda(T)$ possibilities for $|m|$. Therefore

$$
I_{0} \leq \sum_{T / 2 \leq \sqrt{k} \leq T} r_{3}(k) \sum_{|k-l| \leq 5 \lambda(T)} r_{3}(l)
$$

where $r_{3}(k)=\#\left\{n \in \mathbb{Z}^{3}:|n|^{2}=k\right\}$, and

$$
I_{0} \leq c \lambda(T) \sum_{k \leq(2 T)^{2}} r_{3}(k)^{2}
$$

(use $\left.r_{3}(k) r_{3}(l) \leq r_{3}(k)^{2}+r_{3}(l)^{2}\right)$. Since

$$
\lim _{N \rightarrow \infty} N^{-2} \sum_{k \leq N} r_{3}(k)^{2}=K_{0}=\frac{16}{7} \pi^{2} \frac{\zeta(2)}{\zeta(3)}
$$


(see the next section) we obtain

$$
I_{0} \leq c \lambda(T) T^{4} .
$$

Applying this estimate for $T:=2 T, T, T / 2, T / 4, \ldots$, we obtain (by (1.15))

$$
I \leq c \lambda(T)^{2}(\log T)^{-1},
$$

and Lemma 1.2 follows (use the second limit in (1.11)).

Proof of Lemma 1.3. Let

$$
I=\left.\left.(T \log T)^{-1} \int_{1}^{T}\left|\sum_{n}^{\prime} \varphi(2 \pi n \delta)\right| n\right|^{-2} \cos (2 \pi|n| R)\right|^{2} d R .
$$

We will prove that

$$
\lim _{T \rightarrow \infty} I=K_{1}=\frac{32}{7} \pi^{2} \frac{\zeta(2)}{\zeta(3)} .
$$

Then by (1.12), Lemma 1.3 will follow with $K=\pi^{-2} K_{1}$.

Since $\varphi(\xi)$ is isotropic, $\varphi(\xi)=\varphi_{0}(|\xi|)$,

$$
I=(T \log T)^{-1} \int_{1}^{T}\left|\sum_{k=1}^{\infty} r_{3}(k) k^{-1} \varphi_{0}(2 \pi \sqrt{k} \delta) \cos (2 \pi \sqrt{k} R)\right|^{2} d R .
$$

Hence

$$
I=\sum_{k, l=1}^{\infty} r_{3}(k) r_{3}(l) k^{-1} l^{-1} \varphi_{0}(2 \pi \sqrt{k} \delta) \varphi_{0}(2 \pi \sqrt{l} \delta) A(k, l)
$$

with

$$
A(k, l)=(T \log T)^{-1} \int_{1}^{T} \cos (2 \pi \sqrt{k} R) \cos (2 \pi \sqrt{l} R) d R .
$$

Since

$$
A(k, k)=(1 / 2)(\log T)^{-1}\left(1+O\left(T^{-1}\right)\right), \quad T \rightarrow \infty,
$$

the diagonal contribution to $I$ is

$$
I_{\text {diag }}=\sum_{k=1}^{\infty} r_{3}(k)^{2} k^{-2} \varphi_{0}(2 \pi \sqrt{k} \delta)^{2}\left((1 / 2)(\log T)^{-1}+O\left((T \log T)^{-1}\right)\right) .
$$

Since $\varphi_{0}(2 \pi \sqrt{k} \delta)$ produces a smooth cutoff at the scale $\delta^{-2}$ and

$$
\sum_{k=1}^{N} r_{3}(k)^{2} k^{-2}=K_{1}(\log N)(1+o(1)), \quad N \rightarrow \infty,
$$

(see the next section), we obtain

$$
\begin{aligned}
I_{\text {diag }} & =K_{1}\left(\log T^{2}\right)(1 / 2)(\log T)^{-1}(1+o(1)) \\
& =K_{1}(1+o(1)), \quad T \rightarrow \infty
\end{aligned}
$$


For $k \neq l$,

$$
|A(k, l)| \leq c(T \log T)^{-1}|\sqrt{k}-\sqrt{l}|^{-1},
$$

so the off-diagonal contribution to $I$ is estimated as

$\left|I_{\mathrm{off}}\right| \leq \sum_{k \neq l} r_{3}(k) r_{3}(l) k^{-1} l^{-1}\left|\varphi_{0}(2 \pi \sqrt{k} \delta) \varphi_{0}(2 \pi \sqrt{l} \delta)\right|(T \log T)^{-1}|\sqrt{k}-\sqrt{l}|^{-1}$.

By symmetry we may consider only $l>k$. For any $p \geq 1$, consider the block $I_{\text {off }}(p)$ in $I_{\text {off }}$ with $2^{p} \leq k \leq 2^{p+1}$ and $0<j \equiv l-k \leq 2^{p}$. Since $\left|\varphi_{0}(2 \pi \sqrt{k} \delta)\right| \leq c\left(1+k \delta^{2}\right)^{-3}$, this block is estimated as

$$
\begin{gathered}
\left|I_{\text {off }}(p)\right| \leq c\left(1+2^{p} \delta^{2}\right)^{-3} 2^{-2 p} \sum_{k=2^{p}}^{2^{p+1}} \sum_{j=1}^{2^{p}} r_{3}(k) r_{3}(k+j)(T \log T)^{-1} 2^{p / 2} j^{-1} \\
\text { (use } \left.\sqrt{k+j}-\sqrt{k}=j(\sqrt{k+j}+\sqrt{k})^{-1} \geq c j 2^{-p / 2}\right) \text {. Now, } \\
r_{3}(k) r_{3}(k+j) \leq r_{3}(k)^{2}+r_{3}(k+j)^{2}
\end{gathered}
$$

and by (1.16),

hence

$$
\sum_{k=2^{p}}^{2^{p+1}} r_{3}(k+j)^{2} \leq c 2^{2 p}
$$

$$
\begin{aligned}
\left|I_{\mathrm{off}}(p)\right| & \leq c\left(1+2^{p} \delta^{2}\right)^{-3}(T \log T)^{-1} 2^{p / 2} \sum_{j=1}^{2^{p}} j^{-1} \\
& \leq c_{0}\left(1+2^{p} \delta^{2}\right)^{-3}(T \log T)^{-1} 2^{p / 2} p .
\end{aligned}
$$

Consider now the block $I_{\text {off }}(p, q)$ in $I_{\text {off }}$ with $2^{p} \leq k \leq 2^{p+1}$ and $2^{p+q} \leq j \equiv$ $l-k \leq 2^{p+q+1}$, where $p \geq 1$ and $q \geq 0$. This block is estimated as

$$
\begin{aligned}
\left|I_{\text {off }}(p, q)\right| \leq & c\left(1+2^{p} \delta^{2}\right)^{-3} 2^{-p} 2^{-2 p-2 q}(T \log T)^{-1} 2^{p / 2} \\
& \times \sum_{k=2^{p}}^{2^{p+1}} \sum_{j=2^{p+q}}^{2^{p+q+1}} r_{3}(k) r_{3}(k+j) .
\end{aligned}
$$

Since

$$
\sum_{k \leq N} r_{3}(k)=\frac{4 \pi}{3} N^{3 / 2}(1+o(1))
$$

we have

$$
\sum_{j=2^{p+q}}^{2^{p+q+1}} r_{3}(k+j) \leq c 2^{(3 / 2)(p+q)} \quad \text { and } \quad \sum_{k=2^{p}}^{2^{p+1}} r_{3}(k) \leq c 2^{(3 / 2) p},
$$

and thus

$$
\left|I_{\text {off }}(p, q)\right| \leq c\left(1+2^{p} \delta^{2}\right)^{-3} 2^{-q / 2}(T \log T)^{-1} 2^{p / 2}
$$


Combining this estimate with (1.20) we obtain

$$
\left|I_{\mathrm{off}}(p)\right|+\sum_{q=0}^{\infty}\left|I_{\mathrm{off}}(p, q)\right| \leq c\left(1+2^{p} \delta^{2}\right)^{-3}(T \log T)^{-1} 2^{p / 2} p,
$$

and summing now in $p=1,2, \ldots$ we arrive at $\left|I_{\text {off }}\right| \leq c(T \log T)^{-1} \delta^{-1}|\log \delta|$. Since $\delta=T^{-1} \lambda(T)$, this implies $\left|I_{\text {off }}\right| \leq c \lambda(T)^{-1}$. Hence $\lim _{T \rightarrow \infty} I_{\text {off }}=0$ and

$$
\lim _{T \rightarrow \infty} I=\lim _{T \rightarrow \infty} I_{\text {diag }}=K_{1}
$$

(use (1.19)), which proves Lemma 1.3.

2. Evaluation of $N^{-2} \sum_{k=1}^{N}\left|r_{3}(k)\right|^{2}$. Let $\theta(z)=\sum_{n=-\infty}^{\infty} z^{n^{2}}$. Then

$$
S \equiv(2 \pi i)^{-1} \int_{|z|=e^{-2 \pi \delta}} \theta^{3}(z) \overline{\theta^{3}(z)}(d z / z)=\sum_{k=0}^{\infty} e^{-4 \pi k \delta}\left|r_{3}(k)\right|^{2} .
$$

Assuming $\delta \rightarrow 0$ we use the singular series of Hardy (see, e.g., [Gro] or [Vau]). We have (see, e.g., [Gro, p. 151])

$$
\theta\left(e^{2 \pi i h / k-2 \pi z}\right)=(k \sqrt{2 z})^{-1} G(h, k)+\ldots,
$$

hence

$$
\left|\theta\left(e^{2 \pi i h / k-2 \pi z}\right)\right|^{6}=\left(8 k^{6}|z|^{3}\right)^{-1}|G(h, k)|^{6}+\ldots,
$$

where $G(h, k)=\sum_{j=0}^{k-1} e^{2 \pi i h j^{2} / k}$ is the Gaussian sum. Now,

$$
\begin{aligned}
(2 \pi)^{-1} \int\left|\theta\left(e^{2 \pi i h / k-2 \pi(\delta-i(\xi / 2 \pi))}\right)\right|^{6} d \xi & \\
& =\left(8 k^{6}\right)^{-1}|G(h, k)|^{6} \int|\delta-i \xi|^{-3} d \xi+\ldots \\
& =\left(8 k^{6}\right)^{-1}|G(h, k)|^{6} \delta^{-2} \int_{-\infty}^{\infty}\left(x^{2}+1\right)^{-3 / 2} d x+\ldots \\
& =\delta^{-2}\left(4 k^{6}\right)^{-1}|G(h, k)|^{6}+\ldots
\end{aligned}
$$

hence

$$
S=(2 \delta)^{-2} \sum_{h, k} k^{-6}|G(h, k)|^{6}+\ldots,
$$

so that

$$
S=(2 \delta)^{-2} \sum_{k=1}^{\infty} A_{k}+\ldots
$$


with

$$
A_{k}=k^{-6} \sum_{h \bmod k ;(h, k)=1}|G(h, k)|^{6} .
$$

The dots in (2.1) stand for $o\left(\delta^{-2}\right), \delta \rightarrow 0$.

$A_{k}$ is a multiplicative arithmetical function (see [Gro, p. 156]), so that $A_{k_{1} k_{2}}=A_{k_{1}} A_{k_{2}}$ when $\left(k_{1}, k_{2}\right)=1$. Hence

$$
\sum_{k=1}^{\infty} A_{k}=\prod_{p}\left(1+A_{p}+A_{p^{2}}+\ldots\right)
$$

with the product over primes. Now, $A_{2}=0$ and for $a>1,\left|G\left(h, 2^{a}\right)\right|=$ $2^{(a+1) / 2}$ (see [Gro, p. 138]), hence $A_{2^{a}}=2^{-6 a} 2^{3(a+1)} 2^{a-1}=2^{-2 a+2}, a>1$, and

$$
1+A_{2}+A_{4}+A_{8}+\ldots=1+1 / 3=4 / 3 .
$$

When $p>2,\left|G\left(h, p^{a}\right)\right|=\left|\left(h / p^{a}\right)\right|\left|G\left(1, p^{a}\right)\right|=p^{a / 2}$ (see [Gro, p. 138]), hence $A_{p^{a}}=p^{-6 a} p^{3 a} \sum_{h} 1=(p-1) p^{-2 a-1}$ and

$$
1+A_{p}+A_{p^{2}}+\ldots=1+(p(p+1))^{-1} .
$$

Therefore from (2.1) and (2.2),

$S=(2 \delta)^{-2} \frac{4}{3} \prod_{p>2}\left\{1+(p(p+1))^{-1}\right\}+\ldots=\delta^{-2} \frac{2}{7} \prod_{p}\left\{1+(p(p+1))^{-1}\right\}+\ldots$

Now, $\prod_{p}\left\{1+(p(p+1))^{-1}\right\}=\zeta(2) / \zeta(3)$, hence

$$
S=\delta^{-2} \frac{2}{7} \frac{\zeta(2)}{\zeta(3)}+\ldots
$$

Thus

$$
\lim _{\delta \rightarrow 0} \delta^{2} \sum_{k=1}^{\infty}\left|r_{3}(k)\right|^{2} \exp (-4 \pi k \delta)=\frac{2}{7} \frac{\zeta(2)}{\zeta(3)}
$$

and so

$$
\lim _{\delta \rightarrow 0} \delta^{2} \sum_{k=1}^{\infty}\left|r_{3}(k)\right|^{2} \exp (-k \delta)=\frac{32}{7} \pi^{2} \frac{\zeta(2)}{\zeta(3)} .
$$

By the tauberian theorem of Hardy and Littlewood [HL] this implies that

$$
\lim _{N \rightarrow \infty} N^{-2} \sum_{k=1}^{N}\left|r_{3}(k)\right|^{2}=\frac{16}{7} \pi^{2} \frac{\zeta(2)}{\zeta(3)} .
$$

Hence by partial summation,

$$
\lim _{N \rightarrow \infty}(\log N)^{-1} \sum_{k=1}^{N} \frac{\left|r_{3}(k)\right|^{2}}{k^{2}}=\frac{32}{7} \pi^{2} \frac{\zeta(2)}{\zeta(3)} .
$$


Acknowledgements. The authors thank Fernando Chamizo and Henryk Iwaniec for useful remarks and some references. The work is supported by a grant from the Ambrose Monell Foundation and by a grant in aid \# DE-FG02-90ER40542 from the US Department of Energy.

\section{References}

[AP] S. D. Adhikari and Y.-F. S. Pétermann, Lattice points in ellipsoids, Acta Arith. 59 (1991), 329-338.

[Ble1] P. M. Bleher, On the distribution of the number of lattice points inside a family of convex ovals, Duke Math. J. 67 (1992), 461-481.

[Ble2] -, Distribution of energy levels of a quantum free particle on a surface of revolution, ibid. 74 (1994), 45-93.

[BCDL] P. M. Bleher, Z. Cheng, F. J. Dyson and J. L. Lebowitz, Distribution of the error term for the number of lattice points inside a shifted circle, Comm. Math. Phys. 154 (1993), 433-469.

[BK] M. N. Bleicher and M. I. Knopp, Lattice points in a sphere, Acta Arith. 10 (1965), 369-376.

[CI] F. Chamizo and H. Iwaniec, A 3-dimensional lattice point problem, in preparation.

[CN] K. Chandrasekharan and R. Narasimhan, Hecke's functional equation and the average order of arithmetical functions, Acta Arith. 6 (1961), 487-503.

[Che] J.-R. Chen, Improvement on the asymptotic formulas for the number of lattice points in a region of the three dimensions (II), Sci. Sinica 12 (1963), 751-764.

[Cra] H. Cramér, Über zwei Sätze von Herrn G. H. Hardy, Math. Z. 15 (1922), 201-210.

[Gro] E. Grosswald, Representations of Integers as Sums of Squares, Springer, New York, 1985.

[HL] G. H. Hardy and J. E. Littlewood, Tauberian theorems concerning power series and Dirichlet series whose coefficients are positive, Proc. London Math. Soc. 13 (1914), 174.

[H-B] D. R. Heath-Brown, The distribution and moments of the error term in the Dirichlet divisor problem, Acta Arith. 60 (1992), 389-415.

[KN] E. Krätzel and W. G. Nowak, Lattice points in large convex bodies, II, ibid. 62 (1992), 285-295.

[Lan1] E. Landau, Vorlesungen über Zahlentheorie, V. 1, Hirzel, Leipzig, 1927.

[Lan2] -, Ausgewählte Abhandlungen zur Gitterpunktlehre, A. Walfisz (ed.), Deutscher Verlag der Wiss., Berlin, 1962.

[Now] W. G. Nowak, On the lattice rest of a convex body in $\mathbb{R}^{s}$, II, Arch. Math. (Basel) 47 (1986), 232-237.

[Ran] B. Randol, A lattice point problem, I, II, Trans. Amer. Math. Soc. 121 (1966), 257-268; 125 (1966), 101-113.

[Sze] G. Szegö, Beiträge zur Theorie der Laguerreschen Polynome. II: Zahlentheoretische Anwendungen, Math. Z. 25 (1926), 388-404.

[Vau] R. C. Vaughan, The Hardy-Littlewood Method, Cambridge University Press, Cambridge, 1981.

[Vin1] I. M. Vinogradov, On the number of integral points in a given domain, Izv. Akad. Nauk SSSR Ser. Mat. 24 (1960), 777-786. 
[Vin2] I. M. Vinogradov, On the number of integral points in a sphere, ibid. 27 (1963), 957-968.

[Wal] A. Walfisz, Gitterpunkte in mehrdimensionalen Kugeln, PWN, Warszawa, 1957.

DEPARTMENT OF MATHEMATICAL SCIENCES

INDIANA UNIVERSITY-

PURDUE UNIVERSITY INDIANAPOLIS

402 BLACKFORD STREET

INDIANAPOLIS, INDIANA 46202-3272

U.S.A.

E-mail: BLEHER@MATH.IUPUI.EDU
SCHOOL OF NATURAL SCIENCES INSTITUTE FOR ADVANCED STUDY PRINCETON, NEW JERSEY 08540 U.S.A.

Received on 12.3.1994

and in revised form on 25.4.1994 